\title{
Exercise induced release of von Willebrand factor: evidence for hypoxic reperfusion microvascular injury in rheumatoid arthritis
}

Adrian J Farrell, Richard B Williams, Cliff R Stevens, Andrew S Lawrie, Nigel L Cox, David R Blake

\begin{abstract}
Experimental evidence suggests that rheumatoid synovitis may be perpetuated by the generation of reactive oxygen species during hypoxic reperfusion injury. The latter occurs because increased intra-articular pressure during exercise exceeds synovial capillary perfusion pressure, impairing blood flow. The object of this study was to establish a marker for and the mechanism of synovial hypoxic reperfusion injury.

Von Willebrand factor ( $v W F$ ) is only released from endothelial cells and platelets and is an in vivo and in vitro marker of endothelial injury. In vivo exercise induced changes in plasma vWF were therefore investigated in patients with rheumatoid arthritis (RA) compared with controls and in vitro vWF release by human umbilical vein endothelial cells subjected to hypoxiareperfusion. Pre-exercise plasma vWF levels were 1001 and $817 \mathrm{IU} / \mathrm{l}$, increasing after exercise to 1658 and $845 \mathrm{IU} / 1$ in patients with RA and controls respectively. Von Willebrand factor release from human umbilical vein endothelial cells followed a biphasic pattern, occurring during both hypoxia and reperfusion.

Hypoxia reperfusion induced $v W F$ release by human umbilical vein endothelial cells in vitro suggests that exercise induced vWF release in patients with $R A$ is best explained by synovial hypoxic reperfusion injury. This study supports evidence that generation of reactive oxygen species plays a principal part in synovial hypoxic reperfusion injury and suggests $\mathrm{VWF}$ as a useful marker of this phenomenon.
\end{abstract}

\section{(Ann Rheum Dis 1992; 51: 1117-1122)}

Von Willebrand factor (vWF) is a 220 kilodalton glycoprotein produced by endothelial cells and platelet precursors which in plasma exists as a series of multimers up to at least 20000 kilodaltons. Its chief function is the initiation of platelet adhesion to subendothelium at sites of vascular injury." Von Willebrand factor is released from endothelial cells by a $\mathrm{Ca}^{2+}$ dependent mechanism ${ }^{2}$ following a variety of stimuli including oxidant stress. $^{3}$ Several studies have shown increased levels of vWF in the presence of vasculitis; however, levels of vWF are not increased in uncomplicated rheumatoid synovitis. ${ }^{45}$

Experimental evidence suggests that synovitis may be perpetuated by oxidatively driven hypoxic reperfusion injury. ${ }^{6}$ This phenomenon depends on the intra-articular pressure during exercise increasing above the synovial capillary perfusion pressure resulting in the temporary cessation of blood flow. Following this ischaemic episode, reperfusion injury follows, driven by reactive oxygen species. Such events would explain the early clinical response of acute rheumatoid arthritis (RA) to bed rest which would suppress hypoxic and reactive oxygen species induced injury. Conversely, they might also account for the beneficial effects accruing from several months of graded exercise in patients with RA. $^{78}$ Production of reactive oxygen species during exercise may mimic the cytotoxic and antiproliferative effects of gold and D-penicillamine on endothelial cells in vitro. ${ }^{10}$ Gold cytotoxicity appears to be the result of a shift in the ratio of the xanthine dehydrogenase oxidase system favouring the production of reactive oxygen species. ${ }^{11}$ Similarly the antiproliferative effects of D-penicillamine on endothelial cells are $\mathrm{Cu}^{2+}$ dependent and inhibited by catalase, implying that they are mediated by reactive oxygen species derived from hydrogen peroxide. ${ }^{10}$ The suggested common pathway for drugs and exercise is that by impairing microvascular function they cause involution of inflamed synovium, thereby achieving a polyarticular 'autosynovectomy'.

We proposed that $\mathrm{vWF}$ might be an in vivo marker of hypoxic reperfusion injury during joint exercise. To test this hypothesis we measured levels of vWF and its multimeric distribution before and after a standard exercise protocol in patients with active RA and healthy controls, and release of vWF from human umbilical vein endothelial cells subjected to hypoxia and reperfusion. Von Willebrand factor multimers were measured in an attempt to differentiate whether changes in vWF were due to constitutive or secretagogue induced release from Weibel-Palade bodies. The latter contain the higher molecular weight vWF multimers released by secretagogues whereas lower weight vWF multimers are constitutively released.

\section{Patients and methods}

CLINICAL PROTOCOL

The subjects were 12 patients with RA (two men and 10 women, aged 30-79 years) and seven healthy normal controls (three men and four women, aged 32-61 years). The patients were admitted to hospital with active disease which included prominent knee synovitis and all satisfied the 1987 American Rheumatism Association criteria for RA. The two groups conformed to the protocol outlined in the following. Minimum physical activity was 
specified before the study. Subjects rested supine for at least 45 minutes before plasma sampling. Samples were drawn via a $1.4 \mathrm{~mm}$ (17 gauge) plastic cannula at five and ten minutes and immediately before exercise. All patients and controls performed a standard exercise of walking for 15 minutes 'at their own pace' after which further samples were drawn immediately, five, $10,15,20,30,45$, and 60 minutes after exercise. Cannula patency was maintained with normal saline, an initial 1-2 $\mathrm{ml}$ sample being rejected. A fresh syringe was used to aspirate a further sample of which $4.5 \mathrm{ml}$ was taken into $0.5 \mathrm{ml}$ of an antiproteolytic mixture (consisting of $3 \%$ sodium citrate, $10 \mathrm{mM}$ EDTA, $1 \mathrm{mM}$ leupeptin, $6 \mathrm{mM} N$-ethylmaleimide). Sampling was achieved without using venostasis or, if required, two minutes after tourniquet release. Plasma samples were stored on ice until centrifuged at $5^{\circ} \mathrm{C}$ for 10 minutes at $2000 \mathrm{~g}$. Aliquoted plasma was stored at $-70^{\circ} \mathrm{C}$. The study was performed in accordance with the requirements of the Helsinki declaration and the relevant local ethical committees.

\section{HUMAN UMBILICAL VEIN ENDOTHELIAL CELLS SUBJECTED TO HYPOXIA REPERFUSION}

Experiments on the release of vWF from human umbilical vein endothelial cells were performed in passage 2 cells on 96 well tissue culture plates (Costar, Cambridge, MA, USA). The hypoxia chamber used was a desiccator jar with a wire mesh stage perfused with $95 \%$ nitrogen $/ 5 \%$ carbon dioxide. This device yielded hypoxia of the media within the wells to the extent of $6 \%$ oxygen after 30 minutes and $3 \%$ oxygen after 60 minutes. Twenty four hours before the experiment $220 \mu \mathrm{l}$ of medium (E199 with $20 \%$ fetal calf serum was used in all cultures and experiments) was added to each well replacing that in which the cells became confluent. At the start of the experiment half of the medium from each well was removed to measure the baseline level of $\mathrm{vWF}$. The experimental plates were then placed in the hypoxia chamber for periods of $30-60$ minutes at the end of which the medium was sampled. Plates were next placed in $95 \%$ air $/ 5 \%$ carbon dioxide and the medium sampled from a parallel well after $\mathbf{4 0}$ minutes of 'reperfusion'. Control plates were kept in $95 \%$ air $/ 5 \%$ carbon dioxide and sampled after a similar duration to those subjected to hypoxia and reperfusion phases. For experimental and control plates levels of vWF were on average from eight wells.

\section{ESTIMATIONS OF VON WILLEBRAND FACTOR}

Levels of vWF were determined by a double antibody sandwich enzyme linked immunosorbent assay (ELISA). ${ }^{12}$ (The abbreviation vWF refers to von Willebrand factor or immunogenically determined von Willebrand factor levels, i.e. vWF:Ag previously termed factor VIII related antigen or FVIII:RAg). Rabbit antihuman vWF $(100 \mu l)$ (Dako Ltd, High Wycombe, Bucks, United Kingdom), diluted 1:1000 in $0.05 \mathrm{M}$ carbonate buffer (pH 9.6), was added to each well of a 96 well microtitre plate (Dynatech Laboratories, Billinghurst, Sussex, United Kingdom) and incubated for one hour at room temperature in a moist chamber. Plates were then washed three times with $0.05 \%$ Tween 20 in $0.02 \mathrm{M}$ borate buffered

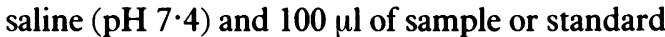
was added to each well and incubated for one hour at room temperature. The plate was again washed three times with $0.05 \%$ Tween 20 in borate buffered saline, and $100 \mu$ peroxidase conjugated rabbit antihuman vWF (Dako) diluted $1: 1000$ in $0 \cdot 1 \%$ Tween 20 in borate buffered saline was added to each well and incubated for one hour. After two washes with $0.05 \%$ Tween 20 in borate buffered saline and one with $0.1 \mathrm{M}$ citrate phosphate buffer $(\mathrm{pH}$ $5 \cdot 0), 100 \mu$ l of substrate was added to each well $(80 \mathrm{mg}$ 1,2-ortho-phenylenediamine dihydrochloride in $15 \mathrm{ml}$ citrate phosphate buffer and $20 \mu \mathrm{l}$ of 30 volumes hydrogen peroxide). After approximately five minutes the reaction was stopped by adding $150 \mu \mathrm{l}$ of $1.0 \mathrm{M}$ sulphuric acid to each well and the absorbance read at 492 nm (Titertek Multiskan MCC, Flow Laboratories, Rickmansworth, United Kingdom). The standard used was a pooled plasma sample, the vWF activity of which was determined against the fourth international vWF standard (NIBSC, South Mimms). Actual vWF values were the mean of three dilutions per sample performed in duplicate. The intrabatch coefficient of variation of the assay was $9.5 \%$. The interbatch coefficient of variation was $15 \cdot 8 \%$.

\section{VON WILLEBRAND FACTOR MULTIMERS}

Multimeric distribution was determined using a Phast electrophoresis system (Pharmacia, Milton Keynes, United Kingdom). ${ }^{13}$ Plasma samples were diluted $1: 5$ in buffer $(10 \mathrm{mM}$ TRIS $\mathrm{HCl}$ with $2 \%$ sodium dodecyl sulphate. $1 \mathrm{mM}$ EDTA and $0.05 \%$ bromophenol blue, $\mathrm{pH} 8.0$ ) incubated at $60^{\circ} \mathrm{C}$ for 30 minutes and applied to an agarose gel with a $13 \mathrm{~mm}$ stacking zone $(0.8 \%$ agarose in $0.125 \mathrm{M}$ TRIS $\mathrm{HCl}$ with $0 \cdot 1 \%$ sodium dodecyl sulphate, $\mathrm{pH} 6 \cdot 8$ ) and a $37 \mathrm{~mm}$ separation zone $(0.8 \%$ agarose in $0.5 \mathrm{M}$ TRIS $\mathrm{HCl}$ with $0 \cdot 1 \%$ sodium dodecyl sulphate, pH 8.8). After electrophoresis and blotting onto a nitrocellulose membrane the latter was placed in a blocking solution (5\% dried milk) for one hour, washed three times with $0.1 \%$ Tween 20 in borate buffered saline and incubated at room temperature with rabbit antihuman vWF diluted 1:750 in borate buffered saline. After three washes with $0.1 \%$ Tween 20 in borate buffered saline an alkaline phosphatase conjugated swine antirabbit antibody (Dako) diluted 1:750 in borate buffered saline was added. Following incubation at room temperature for two hours, two washes with $0 \cdot 1 \%$ Tween 20 borate buffered saline and one wash with $0.1 \mathrm{M}$ ethanolamine buffer ( $\mathrm{pH} 9.6$ ), substrate was poured on and gently mixed $(5 \mathrm{ml}$ nitro blue tetrazolium $0.1 \%$ in ethanolamine buffer, $750 \mu \mathrm{l}$ 10.8 mM 5-bromo-4-chloro-3indolyl phosphate in 2:1 methanol/acetone, 200 $\mu \mathrm{l} 1.0 \mathrm{M}$ magnesium chloride, and $50 \mathrm{~m}$ ethanolamine buffer $\mathrm{pH}$ 9.6). Visualisation occurred within five minutes after which the 
nitrocellulose was washed with distilled water and allowed to dry.

\section{STATISTICS}

Previous studies have shown vWF to be normally distributed in uncomplicated RA. ${ }^{4}$ Data on human umbilical vein endothelial cells were also normally distributed, hence statistical analysis was by Student's $t$ test.

\section{Results}

VON WILLEBRAND FACTOR LEVELS FOLLOWING EXERCISE IN PATIENTS WITH RHEUMATOID ARTHRITIS

There was no difference between mean (SEM) basal levels of vWF in patients (1001 (119) IU/l) and controls (817 (57) IU/l). Controls showed no significant change in levels of $\mathrm{vWF}$ after exercise in either absolute or percentage terms. In patients with RA mean basal levels of vWF (1001 IU/l) increased to 1658 (156) IU/l, which was significant $(p<0.001)$ when compared to controls $(\mathrm{p}<0.01)$ (Table 1$)$. The mean increases in $\mathrm{vWF}$ for patients with RA and controls were $725(128)$ and $27(44)$ IU/l respectively $(p<0.01)$ (fig 1). The mean percentage increases were 79.3 and $3.4 \%$ in patients with RA and controls respectively. The peak levels of $\mathrm{vWF}$ all occurred in the first 20 minutes after exercise,

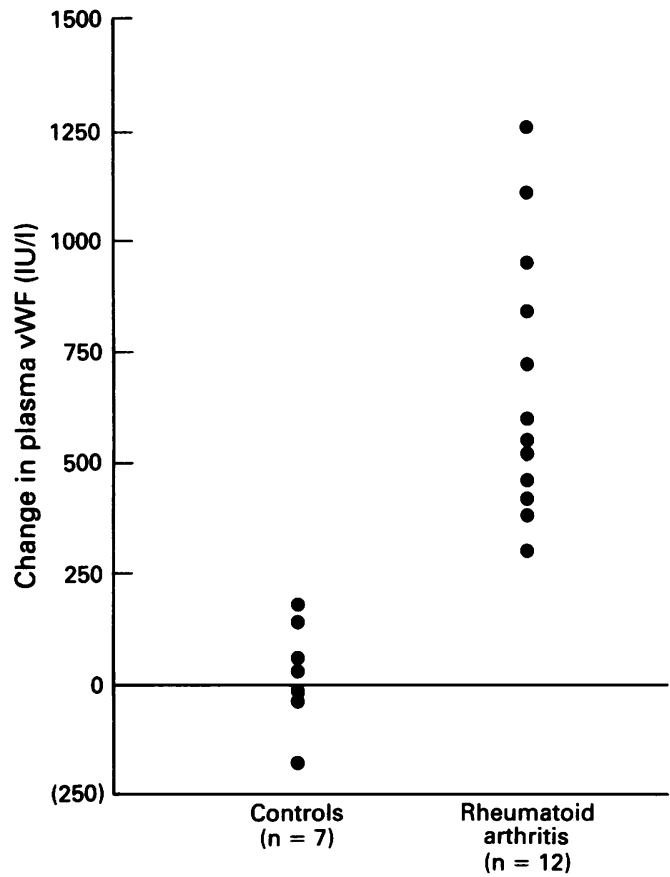

Figure 1 Exercise induced change in plasma von Willebrand factor $(v W F)$ in controls and patients with rheumatoid arthritis.

Table 1 Change in plasma levels of von Willebrand factor (vWF) after exercise in healthv normal controls (mean $(S E M)$ age $47(5 \cdot 2)$ ) and patients with rheumatoid arthritis (mean (SEM) age $62(5 \cdot 4))$

\begin{tabular}{lccc}
\hline & $\begin{array}{l}\text { Mean pre-exercise } \\
v W F(I U / l)\end{array}$ & $\begin{array}{l}\text { Mean post-exercise } \\
v W F(I U / l)\end{array}$ & $\begin{array}{l}\text { Mcan change in } \\
v W F(1 \%)\end{array}$ \\
\hline $\begin{array}{l}\text { Controls }(\mathrm{n}=7) \\
\text { Rheumatoid arthritis }(\mathrm{n}=12)\end{array}$ & $817(57)$ & $845(70)$ & $\begin{array}{c}3 \cdot 4(5 \cdot 0) \\
79 \cdot 3(15 \cdot 9)\end{array}$ \\
\hline
\end{tabular}

p $<0.001$, Student's $t$ test. though in half the subjects the peak level of vWF was immediately after exercise.

There was no change in the distribution of vWF multimers. Although semi-quantitative the technique is routinely applied to the gross changes evident in von Willebrand's disease and should have detected large shifts in multimeric distribution.

RELEASE OF VON WILLEBRAND FACTOR FROM HUMAN UMBILICAL VEIN ENDOTHELIAL CELLS Release of $\mathrm{vWF}$ by human umbilical vein endothelial cells during hypoxia and reperfusion phases was greater than that of cells under control conditions whatever the period of hypoxia (table 2). Baseline levels of vWF were 7.0-9.0 IU/1. After 30 minutes of hypoxia levels of $\mathrm{vWF}$ increased by $2 \cdot 7(0 \cdot 2) \mathrm{IU} / \mathrm{l}$, which was significantly greater than the increase in controls $(1 \cdot 1(0 \cdot 1) \mathrm{IU} / \mathrm{l})(\mathrm{p}<0 \cdot 02)$. There was a

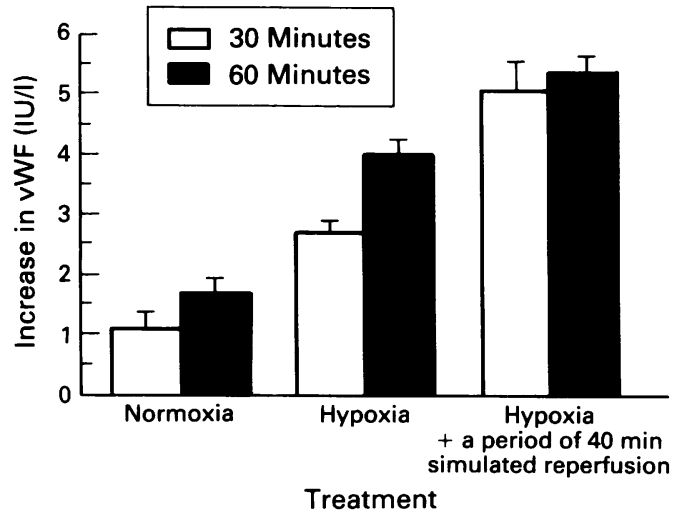

Figure 2 Increase in von Willebrand factor (vWF) levels of human umbilical vein endothelial cell supernatants after periods of 30 or 60 minutes' hypoxia, followed by a standard 40 minutes of simulated reperfusion phase or equivalent normoxic periods for controls.

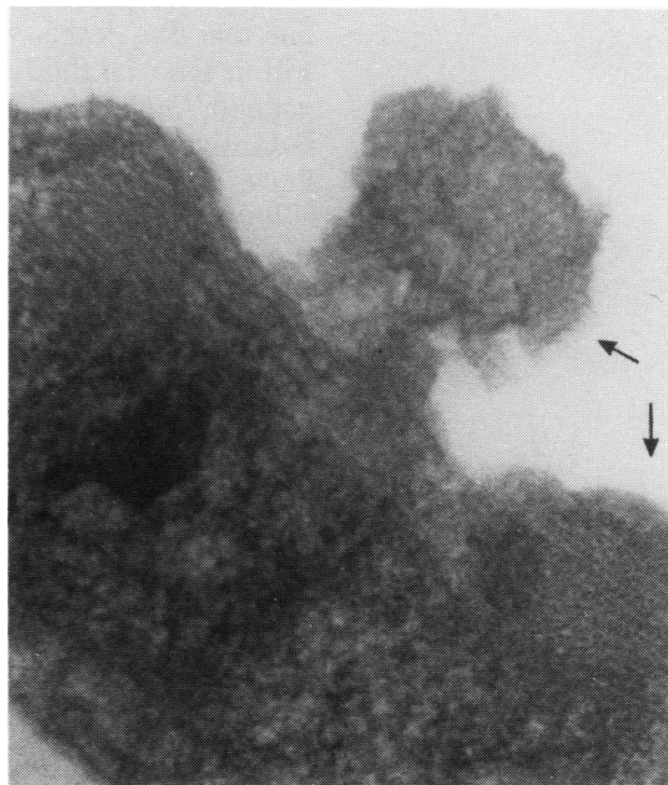

Figure 3 Transmission electron micrograph of human umbilical vein endothelial cell after 30 minutes of hypoxia and 40 minutes of reperfusion. Weibel-Palade bodies (arrows) are seen at and budding away from the luminal membrane. Stained with lead citrate and uranyl acetate. 
Table 2 Increase in levels of von Willebrand factor $(\mathrm{vWF}$ in supernatants human umbilical vein endothelial cells (HUVECS) after various periods of hypoxia, followed by a standard 40 minute reperfusion phase or equivalent normoxic periods for control HUVECS. Values are mean (SEM) levels in IU/l. Baseline levels of vWF in supernatants of HUVECS 7.0-9.0 IU/l

\begin{tabular}{lll}
\hline & \multicolumn{2}{l}{ Duration of hypoxia (minutes) } \\
& 30 & 60 \\
\hline $\begin{array}{l}\text { HUVECS, end of hypoxia } \\
\text { HUVECS, end of hypoxia }\end{array}$ & $2 \cdot 7(0 \cdot 2)$ & $4 \cdot 0(0 \cdot 2)$ \\
$\begin{array}{c}\text { reperfusion } \\
\text { Control HUVECS, end of } \\
\text { equivalent to hypoxia }\end{array}$ & $5 \cdot 1(0 \cdot 5)$ & $5 \cdot 4(0 \cdot 2)$ \\
$\begin{array}{l}\text { Control HUVECS, end } \\
\text { of equivalent to hypoxia } \\
\text { reperfusion }\end{array}$ & $1 \cdot 1(0 \cdot 1)$ & $1 \cdot 7(0 \cdot 2)$ \\
\hline
\end{tabular}

further increase after the subsequent 40 minutes of reperfusion to a total increase of $5 \cdot 1(0.5) \mathrm{IU} / \mathrm{l}$ compared with $1.5(0.2)$ IU/l. Longer periods of hypoxia resulted in proportionately smaller increments in release of VWF but similar final levels of vWF to shorter periods of hypoxia suggesting a limit to the $\mathrm{vWF}$ available for release by human umbilical vein endothelial cells. This also implies an underestimate of reperfusion induced release of vWF. Even so, the rate of increase in $\mathrm{VWF}$ in the reperfusion phase following 30 minutes of hypoxia was far greater than that during hypoxia of 60 minutes' duration. This evidence suggests a biphasic release of $\mathrm{vWF}$ in response to hypoxia reperfusion.

\section{Discussion}

Implicating the vascular system in the pathogenesis of RA has depended on two types of evidence. The description. of synovial microvascular abnormalities, ${ }^{14}$ including those typical of hypoxia, ${ }^{15}$ generally preceded clinical studies of synovial fluid biochemistry. The latter established that the rheumatoid joint was not only hypoxic compared with that in osteoarthritis ${ }^{16}$ but that $\mathrm{Po}_{2}$ levels were less than $15 \mathrm{mmHg}$ within synovial fluid in a significant number of patients ${ }^{17}$ and correlated with other parameters indicative of hypoxia, such as synovial fluid $\mathrm{pH}$, $\mathrm{PCO}_{2}$, and lactate ${ }^{1617}$ as well as microvascular abnormalities on synovial biopsy. ${ }^{18}$ Moreover, the decreased $\mathrm{Po}_{2}$ and $\mathrm{pH}$ of synovial fluid are inversely related to the effusion volume ${ }^{19}$ suggesting a mechanical component to impaired synovial microvasculature function. As a result of increases in intra-articular pressure on load bearing this would be most pronounced on exercise. In some instances intra-articular pressure may exceed systolic pressure and almost invariably synovial capillary perfusion pressure which is estimated at about $30 \mathrm{mmHg}{ }^{20}$ It can be appreciated that whether arguing a primary or secondary pathogenetic role for the microvasculature the interaction between already abnormal vessels further compromised by a mechanically induced cessation of flow may result in a closed and positive feedback loop.

The resurgence of interest in this area owes much to laser Doppler studies which have shown decreased synovial blood flow associated with exercise induced ${ }^{6}$ or simulated increases in intra-articular pressure. ${ }^{21}$ Exercise has been shown to result in synovial fluid hypoxia ${ }^{6}$ and simulations increasing intra-articular pressure lead to increased $\mathrm{PCO}_{2}$ and lactate, and lowered pH. ${ }^{22}$ Similar exercise protocols also showed in vivo damage to biomolecules in synovial fluid ${ }^{6}$ presumed to be secondary to the production of reactive oxygen species on reperfusion of the synovial microvasculature. ${ }^{23}$ This evidence suggests the hypothesis that synovial microvascular dysfunction exacerbated by impaired perfusion, itself the result of increased intraarticular pressure during joint exercise, contributes to the pathogenesis of RA. Laser Doppler studies are, however, limited by their invasive nature, they could theoretically influence the results obtained, and they are not a practical means by which to assess novel therapeutic approaches. Moreover, despite changes in synovial capillary permeability synovial fluid studies are less likely to yield information about endothelial dysfunction as products of endothelial cells tend to be shed into the vessel lumen. Hence there is a need for a marker of synovial endothelial function which could be measured remotely in plasma.

Von Willebrand factor is a reasonable candidate to fulfil these requirements. It is only released by endothelial cells and platelets and can be measured by an established reliable immunoassay. ${ }^{13}$ It is also reportedly increased in a number of clinical disorders characterised by vascular disease. These include renal failure, ${ }^{24}$ diabetic angiopathy, ${ }^{25}$ myocardial infarction, ${ }^{26}$ polyarteritis nodosa, ${ }^{3} 4$ systemic lupus erythematosus, ${ }^{3}$ scleroderma, ${ }^{3}$ Felty's syndrome, ${ }^{4}$ and systemic vasculitis complicating RA. ${ }^{34}$ It also increases during severe infection ${ }^{27}$ and following surgery. ${ }^{427}$ Further evidence that vWF reflects in vivo vascular injury is the fourfold increase in plasma levels of $\mathrm{vWF}$ following endotoxin administration to normal volunteers. ${ }^{28}$ An additional reason for considering vWF suitable in our model is that 'in vitro' oxidant stress ${ }^{5}$ leads to release of vWF by endothelial cells from Weibel-Palade bodies. These specialised organelles store the higher molecular weight vWF multimers which are functionally more effective in initiating platelet adhesion to the subendothelium. ${ }^{29}$ In vitro Weibel-Palade bodies are responsible for the acute release of higher molecular weight vWF multimers in response to secretagogues with lower molecular weight vWF multimers being secreted constitutively. ${ }^{30}$ For this reason we measured the multimeric distribution of $\mathrm{vWF}$ and immunogenic vWF levels.

Basal levels of $v W F$ confirmed previous findings that $\mathrm{VWF}$ is not increased in uncomplicated RA. The data on vWF multimers showed no significant change following exercise. The peak levels of $\mathrm{vWF}$ after exercise in patients with RA all occurred during the first 20 minutes after stopping exercise with the mode immediately after exercise. Levels of $\mathrm{vWF}$ after exercise were unequivocally greater than the levels before exercise in patients with RA and compared with controls. How is this increase in vWF best explained?

Our hypothesis is that during exercise of an 
inflamed joint the intra-articular pressure increases above the synovial capillary perfusion pressure resulting in cessation of blood flow, hypoxia, and reperfusion injury on restoration of flow. Von Willebrand factor should be released from synovial endothelial cells consequent on the initial hypoxic and subsequent oxidant events and act as an easily accessible marker of the event, hypoxia, or oxidation. A crucial question, however, is the extent to which vWF reflects actual endothelial damage. Can we extrapolate that the mechanism of vWF release is also the principal mediator of endothelial injury in the synovial microvasculature?

We have shown vWF release by human umbilical vein endothelial cells during hypoxia and reperfusion, supporting the premise that vWF release in vivo reflects synovial hypoxic reperfusion injury. Direct evidence of the generation of reactive oxygen species during myocardial $^{29}$ and synovial ${ }^{23}$ reperfusion has been obtained by electron spin resonance and spin trapping techniques. The peak generation of reactive oxygen species occurred after two minutes of reperfusion in dogs subjected to 15 minutes of coronary occlusion, ${ }^{30}$ which corresponds with the early increase in VWF levels in our patients after 15 minutes of walking. Moreover, in vitro reactive oxygen species induce human endothelial cells to release $\mathrm{vWF}$ and express GMP-140. ${ }^{3}$ The latter is an endothelial cell membrane associated glycoprotein responsible for polymorphonuclear leucocyte adhesion. Its expression in response to reactive oxygen species clearly suggests increased polymorphonuclear leucocyte adhesion to endothelial cells as a pathway by which reactive oxygen species may initiate or perpetuate inflammatory processes.

This evidence supports the hypothesis that the increases in vWF observed in patients with RA were due to the action of reactive oxygen species on synovial endothelial cells consequent on synovial reperfusion injury and analogous to that observed in the myocardium. Despite peak reactive oxygen species generation occurring early in reperfusion the effects of reactive oxygen species may well be amplified and prolonged via numerous pathways. These may include GMP-140 via increased polymorphonuclear leucocyte adhesion to endothelial cells and local release of vWF into plasma and subendothelium, the latter enhanced by hypoxia induced increases in permeability-the 'trap door' effect. ${ }^{31}$ Clearly vWF may facilitate endothelial injury by promoting platelet adhesion progressing to aggregation and thrombus formation. ${ }^{32}$ Platelet derived growth factor released during the latter is a major stimulus to endothelial injury in atherosclerosis, ${ }^{33}$ amply demonstrated by the resistance of pigs with von Willebrand's disease to atherosclerosis. ${ }^{34}$ Within synovial microvessels, however, release of platelet derived growth factor by aggregating platelets may also stimulate synovial proliferation. Platelet derived growth factor is a potent mitogen to rheumatoid synovial fibroblasts ${ }^{35}$ and in anchorage independent conditions exerts a marked proliferative action in contrast to the minimal effects of interleukin 1, tumour necrosis factor $\alpha$, interferon $\gamma$, transforming growth factor $\beta$, and epidermal growth factor. ${ }^{36}$ Furthermore there is strong evidence to suggest that platelet derived growth factor is the principal mitogen produced by rheumatoid synovium. ${ }^{37}$ The relative importance of platelet versus macrophage derived platelet derived growth factor has yet to be established. Platelet derived growth factor is also a potent vasoconstrictor, thus vWF mediated platelet aggregation at sites of synovial endothelial cell injury could promote hypoxic reperfusion injury directly and indirectly via synovial proliferation. It might be assumed that progression of microvascular injury to occlusion would remove this source of platelet derived growth factor hastening 'autosynovectomy'. A clear correlation between levels of platelet derived growth factor and plasma vWF in patients following myocardial infarction suggests that $\mathrm{vWF}$ also reflects this aspect of endothelial injury. ${ }^{38}$

Extrapolating from this to the clinical situation it is apparent that most patients with RA will in the course of daily life subject their joints to exercise increasing the intra-articular pressure, resulting in repeated hypoxic reperfusion insults to the synovium, creating. a vicious circle and preventing the resolution of microvascular injury and synovial inflammation. The striking clinical response of RA to bedrest accords with this view. Any alternative explanation of our data would require release of $\mathrm{vWF}$ from normoxic vessels beyond the synovial microvasculature. This might occur if platelets or endothelial cells were generally activated in RA, were more sensitive to stimuli releasing vWF, or these stimuli were accentuated by exercise in RA. In vivo the best known stimuli causing $v W F$ release are agents such as adrenaline and vasopressin analogues. The theoretical effect of vasopressor stimuli releasing vWF was mitigated by the protocol stipulating 'walking for 15 minutes at their own pace' thus minimising cardiovascular workload. Moreover, 'steady state' exercise does not increase levels of $\mathrm{vWF}^{37}$ though they do increase in response to maximal exercise or exercise to the point of 'exhaustion'. 3940 Of course, controls performed the exercise for the same duration but far exceeded the workload and distances covered by patients with RA. We cannot, however, exclude a contribution to the observed increase in $\mathrm{VWF}$ due to excessive stimulation or the abnormal responsiveness of endothelium or platelets in RA. Exercise clearly alters levels of vWF and at the synovial microvascular level the consequences of increased platalet aggregation would be similar.

We suggest that the data are most simply explained by radical driven synovial hypoxic reperfusion injury during exercise causing release of $\mathrm{vWF}$ from synovial endothelial cells. Measurement of $\mathrm{vWF}$ should therefore be useful in assessing the modulating actions of drugs such as gold and penicillamine on endothelial cells as well as the development of pharmacological strategies either to limit hypoxic reperfusion injury, or to promote it. Promotion may create an environment that would favour an 'autosynovectomy'. 
We thank Dr P Harrison for his valuable advice. AJF is a Dista Clinical Research Fellow. CRS is supported by the Arthritis and Rheumatism Council. DRB holds an Arthritis and Rheumatism Council endowed Chair at The London Hospital Medical College.

1 Stel H V, Kjell S S, de Groot P G, van Mourik J A, Sixma J J. Von Willebrand factor in the vessel wall mediates platelet adherence. Blood 1985; 65: 85-90.

2 Hamilton $\mathrm{K}$ K, Sims $\mathbf{P}$ J. Changes in cytosolic calcium associated with von Willebrand factor release in human endothelial cells exposed to histamine. 7 Clin Invest 1987; 79: $600-8$

3 Patel K D, Zimmerman G A, Prescott S M, McEver R P, McIntyre T $M$. Oxygen radicals induce human endothelial cells to express GMP-140 and bind neutrophils. 7 Cell Biol 1991; 112: 749-59.

4 Belch J J, Zoma A A, Richards I M, McLaughlin K, Forbe C D, Sturrock R D. Vascular damage and factor VIII $\mathrm{C} D$, Sturrock $\mathrm{R}$ D. Vascular damage and fact
related antigen. Rheumatol Int 1987; 7: 107-11.

5 Woolf A D, Wakerley G, Wallington T B, Scott D G I, Dieppe $P$. Factor VIII related antigen in the assessment of vasculitis. Ann Rheum Dis 1987; 46: 441-7.

6 Blake D R, Merry P, Unsworth J, et al. Hypoxic-reperfusion injury in the inflamed human joint. Lancet 1989; i: 289-93.

7 Harkcom T M, Lampman R M, Banwell B, Castor C W. Therapeutic value of graded aerobic exercise training in rheumatoid arthritis. Arthritis Rheum 1985; 28: 32-9.

8 Lynberg K, Dannieskold-Samsoe B, Halskov $O$. The effect of physical training on patients with rheumatoid arthritis: changes in disease activity, muscle strength and aerobic capacity. Clin Exp Rheum 1988; 6: 253-60.

9 Matsubara $T$, Ziff $M$. Inhibition of human endothelial proliferation by gold compounds. $\mathcal{f}$ Clin Invest 1987; 79: $1440-6$.

10 Matsubara T, Saura R, Hirohata $\mathrm{K}$, Ziff $M$. Inhibition of human endothelial cell proliferation in vitro and neo-
vascularization in vivo by $D$-penicillamine. $\mathcal{f}$ Clin Invest vascularization in

11 Sahinoglu T, Grootveld M, Stevens C R, Thompson S J, Claxson A W D, Blake $D R$. Influence of disodium aurothiomalate on the activities of xanthine dehydrogenase and xanthine oxidase in endothelial cells. Agents Actions 1991; 32: 71-5.

12 Short P E, Williams C E, Picken A M, Hill F G. Factor VIII related antigen: an improved enzyme immunoassay. Med Lab Sci 1982; 39: 351-5.

13 Lawrie A S, Hoser M J, Savidge G F. PHAST assessment of vWF:Ag multimeric distribution. Thromb Res 1990; 59: 369-73.

14 Kulka J P, Bocking P, Ropes M W, Bauer W. Early joint lesions of rheumatoid arthritis. Arch Pathol 1955; 59: 129-50.

15 Kulka J P. The pathogenesis of rheumatoid arthritis. F Chronic Dis 1959; 10: 388-402.

16 Lund-Olesen K. Oxygen tension in synovial fluids. Arthritis Rheum 1970; 13: 769-76.

17 Truehaft R S, McCarty D J. Synovial fluid, pH, lactate, oxygen and carbon dioxide partial pressure in various join diseases. Arthritis Rheum 1971; 14: 475-84

18 Goetzl E J, Falchuk K H, Zeiger L S, et al. A physiological approach to the assessment of disease activity in rheumaapproach to the assessment of disease activity

19 Richman A I, Su E Y, Ho G. Reciprocal relationship of synovial fluid volume and oxygen tension. Arthritis Rheum 1981; 24: 701-5.

20 Williams R B, Carr A, Trimmings N, Blake D R, Cox N L. Measurement of synovial capillary perfusion using video arthroscopy. Br $\mathcal{F}$ Rheumatol 1991; 30 (suppl 1): 39.

21 Geborek P, Forslind K, Wollheim A F. Direct assessment of synovial blood flow and its relationship to induced hydrostatic pressure changes. Ann Rheum Dis 1989; 48: 281-6.
22 James M J, Cleland L G, Rofe A M, Leslie A L. Intraarticular pressure and the relationship between synovial perfusion and metabolic demand. 7 Rheumatol 1990; 17: p21-7.

23 Allen R E, Blake D R, Nazhat N B, Jones P. Superoxide radical generation by inflamed human synovium after radical generation by inflamed

24 Warrell R P, Hultin M B, Coller S B. Increased VIII/von Willebrand factor antigen and von Willebrand factor activity in renal failure. Am $\mathcal{F}$ Med 1979; 66: 226-8.

25 Luftkin E, Fass D, O'Fallow D, Bowie E. Increased von Willebrand's factor in diabetes mellitus. Metabolism 1979; 28: $63-6$.

26 Giustoli R, Musso R, Cacciola E, Cacciola R R, Russo M, Petralito A. Abnormal plasma levels of factor VIII/von Willebrand factor complex in myocardial infarction: expression of acute reaction or index of vascular endothelium damage. Thromb Haemost 1984; 51: 408.

27 Pottinger B E, Read R C, Paleolog E M, Higgins P G, Pearson J D. Von Willebrand factor is an acute phase reactant in man. Thromb Res 1989; 53: 387-94.

28 Gralnick R H, McKeown L P, Wilson O M, Williams S B, Elin $R$ J. Von Willebrand factor release induced by endotoxin 7 Lab Clin Med 1989; 113: 118-22.

$29 \mathrm{Z}$ weier J L. Measurement of superoxide derived free radicals in the reperfused heart: evidence for a free radical mechanism of reperfusion injury. $\mathcal{F}$ Biol Chem 1988; 263 : 1353-7.

30 Bolli R, Patel B S, Jeroudi M O, Lai E K, McCay P B. Demonstration of free radical generation in stunned myocardium of intact dogs with the use of the spin trap al 476-85.

31 Robertson A, Khairallah P. Arterial permeability and vascular disease. The 'trap door' effect. Exp Mol Patho 1973; 18: 241-60.

32 Harker L A, Ross R, Slichter S J, Scott C R. Homocystine induced arteriosclerosis. The role of endothelial injury and platelet response in its genesis. $\mathcal{F}$ Clin Invest 1976; 58 $731-41$.

33 Ross R, Glomset J, Kariya B, Harker L. A platelet dependent serum factor that stimulates the proliferation of arterial smooth muscle cells in vitro. Proc Natl Acad Sci USA 1974; 71: $1207-10$

34 Fuster V, Bowie E J W, Lewis J C, Fass D N, Owen C A Brown A L. Resistance to arteriosclerosis in pigs with von Willebrand's disease. $\mathcal{f}$ Clin Invest 1976; 61: 7222-30.

35 Remmers E F, Lafyatis R, Kumkumian G K, et al. Cytokines and growth regulation of synoviocytes from patients with rheumatoid arthritis and rats with streptococcal cell wall rheumatoid arthritis and rats with streptoco

36 arthritis. Growth Factors 1990; 2: 179-88. $M \mathrm{~B}$, Wilder $\mathrm{R}$ L. Anchorage independent growth of $M$ B, Wilder $R$ L. Anchorage independent growth of
synoviocytes from arthritic and normal joints. Stimulation synoviocytes from arthritic and normal joints. Stimulation by exogenous platelet derived growth factor and inhibition by transforming growth
Invest $1989 ; 83$ : 1267-76.

37 Remmers E F, Sano H, Lafyatis R, et al. Production of platelet derived growth factor $B$ chain mRNA and immunoreactive PDGF B-like polypeptide by rheumatoid synovium: coexpression with heparin binding acidic fibroblas growth factor-1. I Rheumatol 1991; 18: 7-13.

38 Nilsson J, Elgue G, Wallin $M$, Hamsten A, Blombck $M$. Correlation between plasma levels of growth factors and von Willebrand factor. Thromb Res 1989; 54: 125-32.

39 Andrew M, Carter C, O'Brodovich H, Heigenhauser G. Increases in factor VIII complex and fibrinolytic activity Increases in factor VIII complex and fibrinolytic activity 60: 1917-22.

40 Prentice C R M, Forbes C D, Smith S M. Rise of factor VIII after exercise and adrenaline infusion, measured by immunological and biological techniques. Thromb Res 1972; 1: 493-506. 